PREPARED FOR THE U.S. DEPARTMENT OF ENERGY, UNDER CONTRACT DE-AC02-76CH03073

PPPL-3543

PPPL-3543

UC-70

Transport of Energetic lons during Relaxation Oscillations in Plasmas of Spherical Tori

by

Ya.I. Kolesnichenko, V.V. Lutsenko, R.B. White, and Yu.V. Yakovenko

February 2001

NM|

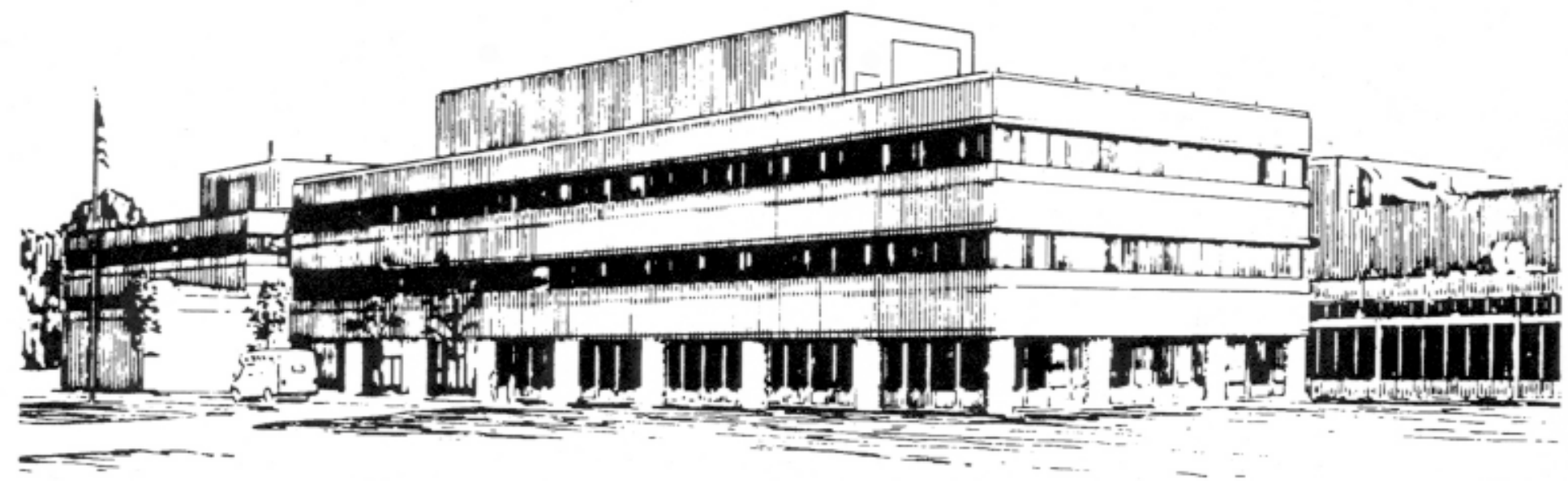

PRINCETON PLASMA PHYSICS LABORATORY PRINCETON UNIVERSITY, PRINCETON, NEW JERSEY 


\section{PPPL Reports Disclaimer}

This report was prepared as an account of work sponsored by an agency of the United States Government. Neither the United States Government nor any agency thereof, nor any of their employees, makes any warranty, express or implied, or assumes any legal liability or responsibility for the accuracy, completeness, or usefulness of any information, apparatus, product, or process disclosed, or represents that its use would not infringe privately owned rights. Reference herein to any specific commercial product, process, or service by trade name, trademark, manufacturer, or otherwise, does not necessarily constitute or imply its endorsement, recommendation, or favoring by the United States Government or any agency thereof. The views and opinions of authors expressed herein do not necessarily state or reflect those of the United States Government or any agency thereof.

\section{Availability}

This report is posted on the U.S. Department of Energy's Princeton Plasma Physics Laboratory Publications and Reports web site in Calendar Year 2001. The home page for PPPL Reports and Publications is: http://www.pppl.gov/pub_report/

DOE and DOE Contractors can obtain copies of this report from:

U.S. Department of Energy

Office of Scientific and Technical Information

DOE Technical Information Services (DTIS)

P.O. Box 62

Oak Ridge, TN 37831

Telephone: (865) 576-8401

Fax: (865) 576-5728

Email: reports@adonis.osti.gov

This report is available to the general public from:

National Technical Information Service

U.S. Department of Commerce

5285 Port Royal Road

Springfield, VA 22161

Telephone: 1-800-553-6847 or

(703) 605-6000

Fax: (703) 321-8547

Internet: http://www.ntis.gov/ordering.htm 


\title{
Transport of energetic ions during relaxation oscillations in plasmas of spherical tori
}

\author{
Ya.I. Kolesnichenko ${ }^{1}$, V.V. Lutsenko ${ }^{1}$, R.B. White $^{2}$, Yu.V. Yakovenko ${ }^{1}$ \\ 1 Scientific Center "Institute for Nuclear Research", Kyiv, 03680, Ukraine \\ 2 Princeton Plasma Physics Laboratory, P.O. Box 451, Princeton, NJ, 08543, USA
}

(February 6, 2001)

\begin{abstract}
It is shown that the confinement of trapped energetic ions in spherical tokamaks during MHD events, such as sawtooth oscillations and internal reconnection events (IRE), essentially depends on $\beta$ ( $\beta$ is the ratio of plasma pressure to the magnetic field pressure). Namely, when $\beta$ is high, the energetic ions are expelled from the plasma core, whereas they are only weakly influenced by the MHD activity at relatively low $\beta$. Because of this, moderate rather than the highest possible $\beta$ may be preferable in spherical tokamaks.
\end{abstract}

Physical processes in toroidal plasmas with low and high $\beta$ may be rather different [1]. Therefore, plasmas in Spherical Tokamaks (ST), where $\beta$ is higher than that in Conventional Tokamaks (CT), may have a number of specific features. In particular, as was shown recently, the fishbone mode associated with trapped particles is stable in high- $\beta$ discharges of STs [2]. In the present work, we consider the influence of high $\beta$ on the confinement of energetic ions in MHD-active plasmas. We assume that the MHD activity is localized in the plasma core and associated with the periodic development of large-scale helical perturbations which are terminated by the reconnection of magnetic field lines (this is presumably the case during, e.g., sawtooth oscillations, internal reconnection events etc.). Such MHD activity manifests itself as relaxation oscillations of plasma parameters and is typical for tokamaks. 
The effect of MHD activity on energetic ions was studied in many works but mainly for CTs. Experiments on CTs have shown that MHD activity can significantly deteriorate confinement of fast ions [3-5]. MHD activity was observed in experiments on the START spherical tokamak [6]. A variety of MHD phenomena was observed in the ohmic heated plasmas in National Spherical Torus Experiment (NSTX), including sawtooth oscillations, internal and global reconnection [7]; NBI experiments including the investigation of the energetic ion behavior started in autumn 2000 [8]. A theoretical study of the effect of sawtooth oscillations on fast ions in CTs resulted in the prediction of the critical energy, $\mathcal{E}_{\text {crit }}$, such that the trapped ions with energy higher than $\mathcal{E}_{\text {crit }}$ are not sensitive to the sawtooth crash (the crash is the relaxation phase of the oscillations), whereas the ions with $\mathcal{E}<\mathcal{E}_{\text {crit }}$ are strongly redistributed by the crash [9]. This prediction was confirmed experimentally [10]. The existence of the critical energy is a consequence of the fact that the precessional motion of particles along the large azimuth of the torus destroys the interaction of ions with magnetic helical perturbations when the ion energy is sufficiently high. Therefore, $\mathcal{E}_{\text {crit }}$ exists for all the MHD events mentioned above. Its magnitude can be evaluated from the condition that the characteristic time of the toroidal precession is equal to the duration of the crash $\left(\tau_{c r}\right)$ as follows:

$$
\mathcal{E}_{\text {crit }}=\left.\frac{2 \pi M k r R_{0} \omega_{B}}{n \tau_{c r}}\right|_{r=r_{s}} \propto \frac{A_{s} B S}{n \tau_{c r}}
$$

where $n$ is the toroidal mode number (which was taken equal to unity in Ref. [9]), $k$ is the elongation of the plasma cross section, $r_{s}$ is the radius of the $q=1$ flux surface, $M$ is the ion mass, $\omega_{B}$ is the cyclotron frequency, $R_{0}$ is the major radius of the torus, $S$ is the square of the $q=1$ flux-surface cross section, $A_{s}=R_{0} / r_{s}$, and $B$ is the magnetic field strength. When $n=1, \tau_{c r} \sim 10^{-4} \mathrm{~s}, \mathcal{E}_{\text {crit }}$ varies in the range $300 \div 700 \mathrm{keV}$ for $\alpha$-particles in TFTR and JET, depending on plasma parameters. Because the product $A_{s} B S$ in STs is relatively small, $\mathcal{E}_{\text {crit }}$ in STs is much less than that in CTs. However, existing theory is relevant for plasmas with low $\beta$, in which the effects of plasma diamagnetism are negligible. In high- $\beta$ plasmas, as we will show in this Letter, trapped ions are attached to evolving flux surfaces 
and, thus, are expelled from the plasma core by crashes of MHD events even when their energy exceeds $\mathcal{E}_{\text {crit }}$.

The difference in the behavior of trapped particles in high- $\beta$ and low- $\beta$ plasmas can be explained as follows. The instabilities causing magnetic reconnection in low- $\beta$ plasmas are essentially Alfvénic in the sense that they weakly perturb the magnetic field strength. Therefore, the particle precession is weakly affected by the perturbations and tends to move particles along unperturbed flux surfaces (i.e., along the flux surfaces that existed before the crash), thus preventing their redistribution. On the other hand, when $\beta$ is so high that $8 \pi \nabla p>B^{2} / R$ ( $p$ is the plasma pressure), the plasma diamagnetism results in a "magnetic valley", i.e., a minimum of the equilibrium magnetic field near the magnetic axis (the magnetic valley exists in, e.g., high- $\beta$ plasma equilibria in NSTX [11]). This reverses the direction of the precession but does not change its character in the sense that it is directed along the large azimuth of the torus, $\phi$. A crash of relaxation oscillations results in a helical displacement of the plasma core and strongly disturbs the plasma pressure, leading to $\nabla \widetilde{p} \sim \nabla p^{0}$, where tilde and the superscript " 0 " denote perturbed and equilibrium quantities, respectively. Because of this the magnetic valley is displaced and split along $\phi$, thus forming several local magnetic wells. This completely changes the character of the particle precessional motion. Namely, as we will show, the precession will result in the particle bounce-averaged orbits that move together with the magnetic wells frozen into the plasma.

Let us proceed to more detailed analysis. We consider the bounce-averaged motion of a particle in an evolving magnetic configuration. We assume that the flux surfaces exist throughout the crash and can be described by a flux function, $\psi$, varying in time. In addition, we assume that the crash duration exceeds the particle bounce period and a particle orbit weakly deviates from a magnetic field line for one bounce. Then the longitudinal adiabatic invariant, $J=M /(2 \pi) \oint d s v_{\|}\left(v_{\|}\right.$is the particle longitudinal velocity, $s$ is the distance along the field line), is approximately conserved. We use flux coordinates $(\psi, \theta, \phi)$, in which the equation of the field line is $\theta=q^{-1}(\psi) \phi+\eta$, where $q$ is the safety factor, $\eta$ is the field line label. Then $J=J(\psi, \eta)$, and equations of the bounce-averaged motion can be written 
as $\dot{\psi}=C_{1} \partial J / \partial \eta, \dot{\eta}=-C_{1} \partial J / \partial \psi$, where $C_{1}$ is a certain function of the metric properties of the magnetic field and the particle pitch angle. For the deeply trapped particles, the derivatives of $J$ are proportional to the corresponding derivatives of $B_{*}$, where $B_{*}(\psi, \eta)=$ $B\left(\psi, \theta\left(\psi, \eta, \phi_{*}\right), \phi_{*}\right)$ is the minimum of the magnetic field on a field line, $\phi_{*}=\phi_{*}(\psi, \eta)$ is the location of the minimum determined by the equations $\partial B / \partial \theta+q \partial B \partial \phi=0$ and $\theta=q^{-1} \phi+\eta$. Taking this into account, we conclude that $B_{*}$ is conserved, i.e., the banana center moves along a trajectory on which the minimum of the magnetic field on the field line does not change. Now we can present the equations of motion of deeply trapped particles as follows:

$$
\dot{\psi}=C_{2} \frac{\partial B}{\partial \theta}, \quad \dot{\eta}=-C_{2}\left(\frac{\partial B}{\partial \psi}-\frac{q^{\prime}}{q^{2}} \phi \frac{\partial B}{\partial \theta}\right)
$$

where the right-hand sides are taken at $\theta=q^{-1} \phi_{*}+\eta, \phi=\phi_{*} ;$ prime denotes $d / d \psi$; $C_{2}=C_{2}(\psi, \eta)$. Assuming that the reconnection process is sufficiently slow for the plasma to be in approximate equilibrium at each moment of time, we use the following Taylor series expansion of the magnetic field strength in the plasma core [12]:

$$
B=B_{0}(\phi)\left\{1+\psi^{1 / 2} \mathcal{A}_{1}(\phi) \cos \left[\theta-\theta_{1}(\phi)\right]+\psi \mathcal{A}_{2}(\phi)+\psi \mathcal{A}_{3}(\phi) \cos \left[2 \theta-2 \theta_{2}(\phi)\right]\right\}
$$

The $\mathcal{A}_{2}(\phi)$ term in Eq. (3) characterizes the growth of the flux-surface-averaged magnetic field with the radius (i.e., the average magnetic well). It can be shown that the increase of the plasma pressure gradient results in the increase of $\mathcal{A}_{2}$ in both axisymmetric tokamak equilibria and stellarator equilibria. If we assume that $\beta$ is high, and the $\mathcal{A}_{2}(\phi)$ term in Eq. (2) dominates, we arrive at the equations $\dot{\psi} \approx 0, \dot{\eta} \approx-C_{2} B_{0}\left(\phi_{*}\right) \mathcal{A}_{2}\left(\phi_{*}\right)$, from which it follows that the particle rotates along a line $\psi=$ const in the $(\psi, \eta)$ plane. The presence of the other terms results only in weak oscillations around this line. This implies that the particle moves approximately along a flux surface. We can conclude from this that when the pressure gradient is sufficiently high, the precession tends to attach particles to evolving flux surfaces, in particular, to magnetic islands formed and moving during the crash of an MHD event. Therefore, in contrast to the case of a low- $\beta$ plasma, the precession in a high- $\beta$ plasma is a factor promoting the redistribution of particles by the crash. 
To evaluate the magnitude of the required $\beta$, we suggest a simple model of plasma equilibrium during the crash. Our starting point is a linear theory which describes the initial stage of a crash. Using the equation of motion of the ideal MHD and assuming that $\omega \ll k_{\perp} v_{A}, k_{\|} \ll k_{\perp}$, and $\mathcal{K} \ll k_{\perp}$ (where $\omega$ is the perturbation frequency, $v_{A}$ is the Alfvén velocity, $\mathcal{K}$ is the field line curvature, $k_{\|}$and $k_{\perp}$ are the longitudinal and perpendicular wave numbers, respectively), we can write [13]:

$$
\widetilde{|B|^{2}}+8 \pi \widetilde{p} \approx 0
$$

Now we construct a model of equilibrium at the nonlinear stage of an MHD event localized inside a certain mixing radius, $r_{m i x}$. At first, we introduce a reference axisymmetric equilibrium. We take it in such a way that it is characterized by $\nabla p_{\text {ref }}=0$ inside the mixing radius, where the subscript "ref" refers to the reference equilibrium. Then we choose $p_{\text {ref }}$ equal to the average pressure in the mixing region before the crash and introduce the flux coordinates $(r, \theta, \phi)$ corresponding to the reference equilibrium. Assuming that $r \ll R_{0}$ for $r<r_{\text {mix }}$, we approximate $B_{\text {ref }}$ at $r<r_{\text {mix }}$ as $B_{\text {ref }}=B_{0} R_{0} / R$, where $R=R_{0}+r \cos \theta$ is the distance to the axis of symmetry. Finally, we assume that approximate helical symmetry is conserved during the $\operatorname{crash}(\psi=\psi(r, \alpha)$ with $\alpha=m \theta-n \phi, m$ is the poloidal mode number $)$ and suppose that

$$
|B|^{2}=B_{\text {ref }}^{2}-8 \pi\left(p-p_{\text {ref }}\right)
$$

for $r<r_{\text {mix }}$, where $p=p[\psi(r, \alpha)]$. Linearizing Eq. (5) near the initial equilibrium, we recover Eq. (4); therefore, the former can be considered as a simple generalization of the latter. Note that the effect of the pressure perturbation on the equilibrium shape is disregarded in Eq. (5). This is justified because the variable part of the pressure $\left(p-p_{\text {ref }}\right)$ responsible for the possible deformation of the equilibrium shape is small.

Substituting Eq. (5) to the expression for the longitudinal adiabatic invariant and assuming that $1-n q / m \ll 1$ (which enables us to replace the integration along a field line by the integration at $r=$ const, $\alpha=$ const), we find: 


$$
J(r, \alpha)=\frac{8 R_{0}}{\pi}\left(\frac{\mu \bar{B} \epsilon}{M}\right)^{1 / 2}\left[\left(\kappa^{2}-1\right) \mathbf{K}(\kappa)+\mathbf{E}(\kappa)\right]
$$

where $\bar{B}=B_{0}\left[1-8 \pi\left(p(r, \alpha)-p_{\text {ref }}\right) / B_{0}^{2}\right]^{1 / 2}, \epsilon=r / R_{0}, \mathbf{K}(\kappa)$ and $\mathbf{E}(\kappa)$ are the complete elliptic integrals of the first and second kind, respectively; $\mu$ is the particle magnetic moment; $\kappa$ is the particle trapping parameter, which depends on $\bar{B}$ and, thus, on the plasma pressure as follows:

$$
\kappa^{2}(r, \alpha)=\frac{\mathcal{E}(r, \alpha)-\mu \bar{B}(r, \alpha)(1-\epsilon)}{2 \mu \bar{B}(r, \alpha) \epsilon}
$$

Note that the assumption $1-n q / m \ll 1$ implies that a perturbation almost does not change $B(s)$ ( $s$ is the distance along a field line), and, thus, the particles remain trapped near the equatorial plane of the torus, where $B(s)$ is minimum. Differentiating Eq. (6) in $r$ and $\alpha$, we obtain the following equations of the bounce-averaged particle motion:

$$
\begin{gathered}
\dot{r}=v_{E}^{1}-\frac{\mu \bar{B}}{M k \omega_{B} R_{0}} \frac{1}{\epsilon} \frac{\partial \bar{B}}{\partial \alpha}, \\
\dot{\alpha}=v_{E}^{2}+\frac{\mu \bar{B}}{M k \omega_{B} R_{0}}\left(\frac{1}{\epsilon} \frac{\partial \bar{B}}{\partial r}-\frac{n G}{r}\right),
\end{gathered}
$$

where $\vec{v}_{E}$ is the velocity of the $\vec{E} \times \vec{B}$ drift, $v_{E}^{1}$ and $v_{E}^{2}$ are its contravariant components in the coordinate system $\left(x^{1}, x^{2}, x^{3}\right)=(r, \alpha, \phi), \vec{E}$ is the electric field associated with the evolution of the magnetic configuration and the concomitant plasma motion; $G=2 \mathbf{E}(\kappa) / \mathbf{K}(\kappa)-1$.

Equations (8), (9) can also be obtained by proceeding from the expression $\vec{v}=$ $\vec{v}_{\|}+\vec{v}_{E}+\vec{v}_{D}$ for the particle velocity with $\vec{v}_{D}$ being the drift velocity given by $\vec{v}_{D}=$ $c /\left(e|B|^{2}\right) \vec{B} \times\left(\mu \nabla B+M v_{\|}^{2} \overrightarrow{\mathcal{K}}\right), e$ being the particle charge. Using the expression $\overrightarrow{\mathcal{K}}=$ $|B|^{-2}\left[\nabla\left(|B|^{2} / 2+4 \pi p\right)-\nabla_{\|}|B|^{2} / 2\right]$, where $\nabla_{\|}=\vec{b}(\vec{b} \cdot \nabla)$, we can express $\nabla B$ in terms of $\nabla p$ and $\overrightarrow{\mathcal{K}}$. Then, if we assume that the magnetic curvature is not affected by the crash and perform bounce averaging, we arrive at Eqs. (8), (9). Thus, the assumption $r / R \ll 1$ made above to construct the equilibrium is not a necessary condition for Eqs. (8), (9) to be valid. Therefore, the derived equations remain valid when perturbations are localized away 
from the magnetic axis, which may be the case during, e.g., IRE. However, they are not applicable when the dominant plasma mixing during MHD events is associated with turbulence (note that small-scale turbulence has little effect on trapped energetic ions $[14,15]$ ).

Let us analyze Eqs. (8), (9). The first terms in these equations describe the $\vec{E} \times \vec{B}$ drift. They are the terms driving the particle redistribution by freezing the particles to the evolving flux surfaces [9]. The conventional (toroidal) precession is described by the last term in Eq. (9). The competition of the conventional precession and the $\vec{E} \times \vec{B}$ drift motion results in $\mathcal{E}_{\text {crit }}$. The other terms in Eqs. (8), (9) are associated with the plasma diamagnetism. Taking into account that $\bar{B}=\bar{B}(p)$ and $p=p(\psi)$, we conclude from Eqs. (8), (9) that the precession associated with the plasma diamagnetism conserves $\psi$, i.e., leads to the particle motion along the flux surfaces rather than along the $r=$ const surfaces. This confirms the conclusion made above with using Eq. (2). Thus, the conventional precession is the only factor preventing the particle redistribution by the crash. The diamagnetic precession overrides the conventional precession and, thus, attaches the particles to the moving flux surfaces when $\partial \ln \bar{B} / \partial \ln r>\epsilon$, which leads to the estimate $\beta\left(r_{s}\right)>\epsilon\left(r_{s}\right)$, where $\beta\left(r_{s}\right) \equiv$ $8 \pi p\left(r_{s}\right) / B_{0}^{2}$. The latter is essentially the condition of the existence of the magnetic valley in the equilibrium magnetic field strength.

It follows from our analysis that in high- $\beta$ plasmas the bounce-averaged orbits of deeply trapped particles with $\mathcal{E}>\mathcal{E}_{\text {crit }}$ approximately follow the level lines of $B_{*}=$ const, which form $n$ families of closed curves in the equatorial plane of the torus outside the magnetic axis (see Fig. 1). When the energy is low, the role of precession is negligible, and the trapped particles move together with the evolving flux surfaces without motion along them. But the final result of the crash from the point of view of the rearrangement of the radial profiles is almost the same for the slow and the fast ions when $\beta$ is high. A possible difference in their redistribution may be associated with the fact that the diamagnetic precession is weakened at the final stage of the crash, when the pressure gradients become small.

In order to demonstrate the dependence of the particle transport on $\beta$, we carry out a numerical simulation of the particle motion during a sawtooth crash. With this purpose 
we use a set of equations consisting of Eqs. (8), (9), [with $v_{E}^{1}=-c\left(B_{0} r k\right)^{-1} \partial \Phi / \partial \alpha, v_{E}^{2}=$ $c\left(B_{0} r k\right)^{-1} \partial \Phi / \partial r, \Phi$ the scalar potential of the perturbation], the equation $J(r, \alpha, \kappa)=$ const (which determines the time dependence of $\kappa$ ), the equation $\vec{E} \cdot \vec{B}=0$ (which connects $\Phi$ and $\psi$ ), and the equations for $\psi(r, \alpha, \phi, t)$ from the crash model of Ref. [9] approximating the Kadomtsev type of the sawtooth crash. As a trapped particle approaching the plasma center may occasionally turn into a passing one, the equations were extended to describe the motion of passing particles (in particular, terms describing the longitudinal motion were added, see Ref. [16] for details). The results of calculations for a well-trapped particle with $\mathcal{E} \gg \mathcal{E}_{\text {crit }}$ are shown in Fig. 2. We observe that the increase of $\beta$ strongly changes the effect of the sawtooth crash on the particle. Namely, the particle, which was not sensitive to the crash at low $\beta$, is expelled from the plasma core in the high- $\beta$ plasma.

In conclusion, we have shown for the first time that when the particle energy exceeds a certain critical magnitude, $\mathcal{E}_{\text {crit }}$ given by Eq. (1), the influence of MHD events accompanied by the helical displacement of the plasma core and reconnection of the magnetic field lines on the energetic ions in high- $\beta$ and low- $\beta$ plasmas may be different. When $\beta$ is high, trapped ions with $\mathcal{E}>\mathcal{E}_{\text {crit }}$ are expelled from the plasma core because of the interaction with the MHD perturbations, in contrast to the low- $\beta$ case. This may take place in high- $\beta$ discharges of STs characterized by the presence of the "valley" in the equilibrium magnetic field. The different influence of the MHD activity on energetic ions in high- $\beta$ and low- $\beta$ plasmas is explained by the fact that when $\beta$ is high, plasma diamagnetism in the presence of large perturbations of plasma pressure changes the character of the particle precessional motion. Namely, it becomes directed along disturbed flux surfaces independent of their form, which leads to the particle redistribution due to the evolution of the magnetic configuration during the crashes of the MHD events. The magnitude of $\mathcal{E}_{\text {crit }}$ in STs is much less than that in CTs, whereas the fraction of trapped particles is larger; therefore, the predicted $\beta$-dependence of transport in MHD-active plasmas may be relevant to a quite considerable fraction of energetic ions. This indicates that moderate $\beta$ rather than the maximal one possible in STs may be preferable. Experimental investigation of the problem as well as 
theoretical study of resonance mechanisms of the particle transport $[17,18]$ are required to draw a final conclusion concerning optimal $\beta$ in STs.

The research described in this work was made possible in part by Award No. UP2-2114 of the U.S. Civilian Research \& Development Foundation, the U.S. Department of Energy under contract number DE-AC02-76-CHO3073, and the International Atomic Energy Agency Research Contract No. 10539. Two of the authors (Ya.K. and Yu.Y.) acknowledge the hospitality of the Princeton Plasma Physics Laboratory. 


\section{REFERENCES}

[1] Yanlin Wu, C.Z. Cheng, and R.B. White, Phys. Plasmas 13369 (1994).

[2] Ya.I. Kolesnichenko, V.V. Lutsenko, and V.S. Marchenko, Phys. Rev. Lett. 82, 3260 (1999).

[3] G.J. Sadler et al., Fusion Technol. 18, 556 (1990).

[4] S.J. Zweben et al., Nucl. Fusion 40, 91 (2000).

[5] G.J. Kramer et al., Nucl. Fusion 40, 1383 (2000).

[6] M. Gryaznevich et al., Phys. Rev. Lett. 80, 3972 (1998).

[7] M.G. Bell for the NSTX Research Team, Physics results from the National Spherical Torus Experiment, Report PPPL-3459, http://www.pppl.gov (2000).

[8] S.M. Kaye et al., Initial physics results from the National Spherical Torus Experiment, Report PPPL-3533, http://www.pppl.gov (2001).

[9] Ya.I. Kolesnichenko and Yu.V. Yakovenko, Nucl. Fusion 36, 159 (1996).

[10] N.N. Gorelenkov et al., Nucl. Fusion 37, 1053 (1997).

[11] D.R. Mikkelsen et al., Phys. Plasmas 4, 3667 (1997).

[12] D.A. Garren and A.H. Boozer, Phys. Fluids B 3, 2805 (1991).

[13] B. Coppi, S. Migliuolo, and F. Porcelli, Phys. Fluids 31, 1630 (1988).

[14] H.E. Mynick and J.A. Krommes, Phys. Fluids 23, 1229 (1980).

[15] G. Manfredi and R.O. Dendy, Phys. Rev. Lett. 76, 4360 (1996).

[16] Ya.I. Kolesnichenko, V.V. Lutsenko, Yu.V. Yakovenko, and G. Kamelander, Phys. Plasmas 4, 2544 (1997).

[17] Ya.I. Kolesnichenko, V.V. Lutsenko, R.B. White, and Yu.V. Yakovenko, Nucl. Fusion 
40, 1325 (2000).

[18] Ya.I. Kolesnichenko, V.V. Lutsenko, R.B. White, and Yu.V. Yakovenko, Phys. Rev. Lett. 84, 2152 (2000). 


\section{FIGURES}

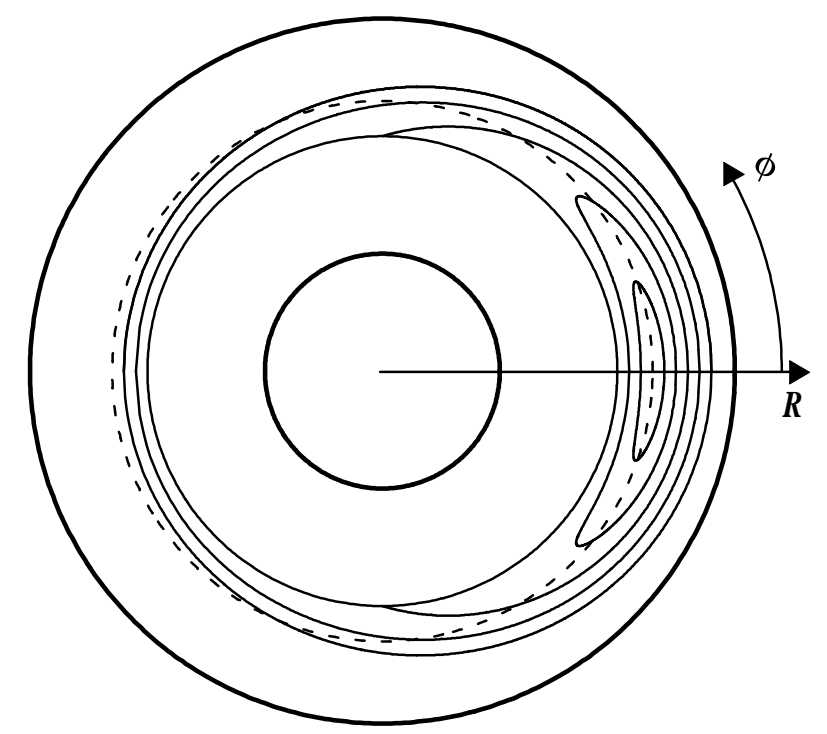

FIG. 1. Sketch of orbits of deeply trapped energetic ion with $\mathcal{E}>\mathcal{E}_{\text {crit }}$ in the equatorial plane of the tokamak when the plasma core is shifted by the $m=n=1$ mode. Solid lines, orbits in the limit case of very high $\beta$ (when they coincide with level lines of the plasma pressure); dashed line, an orbit in the low- $\beta$ case, when the conventional precession dominates; bold lines, the plasma boundaries. 

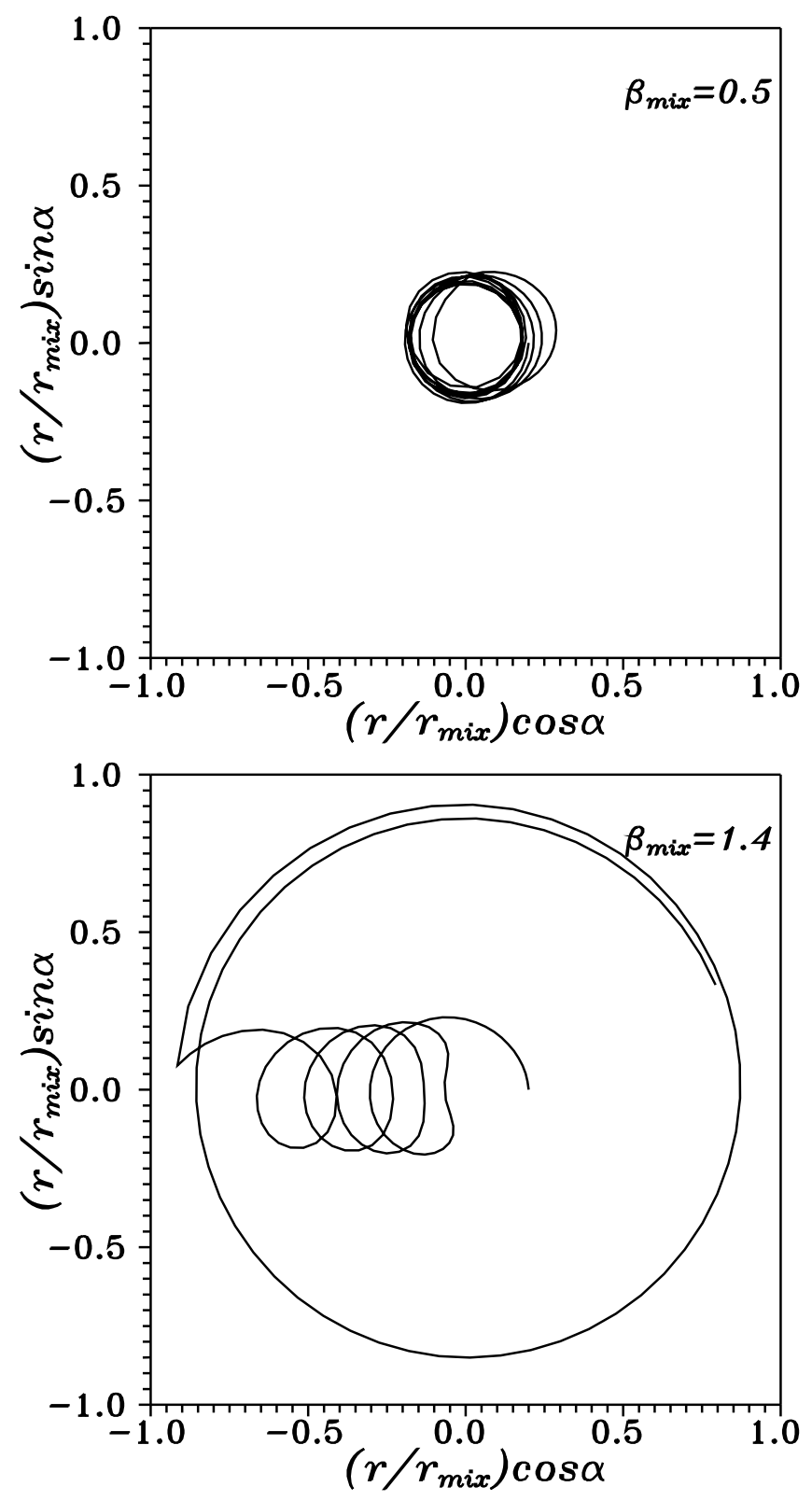

FIG. 2. Motion of trapped energetic ions with $\mathcal{E} \gtrsim \mathcal{E}_{\text {crit }}$ in an NSTX-like tokamak with $r_{m i x} / a=1 / 3$ for various $\beta$ during sawtooth crashes. Notations: $r_{m i x}$ is the sawtooth mixing radius, $\alpha=\theta-\phi, \beta_{\text {mix }}=8 \pi\left[p(0)-p\left(r_{\text {mix }}\right)\right] / B_{0}^{2}, B_{0}$ is the magnetic field on the magnetic axis in the presence of the plasma. We observe that the crash has a strong influence on the ions only when $\beta$ is sufficiently high. 


\section{External Distribution}

Plasma Research Laboratory, Australian National University, Australia

Professor I.R. J ones, Flinders University, Australia

Professor J oão Canalle, Instituto de Fisica DEQ/IF - UERJ , Brazil

Mr. Gerson O. Ludwig, Instituto Nacional de Pesquisas, Brazil

Dr. P.H. Sakanaka, Instituto Fisica, Brazil

The Librarian, Culham Laboratory, England

Library, R61, Rutherford Appleton Laboratory, England

Mrs. S.A. Hutchinson, JET Library, England

Professor M.N. Bussac, Ecole Polytechnique, France

Librarian, Max-Planck-Institut für Plasmaphysik, Germany

J olan Moldvai, Reports Library, MTA KFKI-ATKI, Hungary

Dr. P. Kaw, Institute for Plasma Research, India

Ms. P.J . Pathak, Librarian, Insitute for Plasma Research, India

Ms. Clelia De Palo, Associazione EURATOM-ENEA, I taly

Dr. G. Grosso, Instituto di Fisica del Plasma, Italy

Librarian, Naka Fusion Research Establishment, J AERI, J apan

Library, Plasma Physics Laboratory, Kyoto University, J apan

Research Information Center, National Institute for Fusion Science, J apan

Dr. O. Mitarai, Kyushu Tokai University, J apan

Library, Academia Sinica, Institute of Plasma Physics, People's Republic of China

Shih-Tung Tsai, Institute of Physics, Chinese Academy of Sciences, People's Republic of China

Dr. S. Mirnov, Triniti, Troitsk, Russian Federation, Russia

Dr. V.S. Strelkov, Kurchatov Institute, Russian Federation, Russia

Professor Peter Lukac, Katedra Fyziky Plazmy MFF UK, Mlynska dolina F-2, Komenskeho Univerzita, SK-842 15 Bratislava, Slovakia

Dr. G.S. Lee, Korea Basic Science Institute, South Korea

Mr. Dennis Bruggink, Fusion Library, University of Wisconsin, USA

Institute for Plasma Research, University of Maryland, USA

Librarian, Fusion Energy Division, Oak Ridge National Laboratory, USA

Librarian, Institute of Fusion Studies, University of Texas, USA

Librarian, Magnetic Fusion Program, Lawrence Livermore National Laboratory, USA

Library, General Atomics, USA

Plasma Physics Group, Fusion Energy Research Program, University of California at San Diego, USA

Plasma Physics Library, Columbia University, USA

Alkesh Punjabi, Center for Fusion Research and Training, Hampton University, USA

Dr. W.M. Stacey, Fusion Research Center, Georgia Institute of Technology, USA

Dr. J ohn Willis, U.S. Department of Energy, Office of Fusion Energy Sciences, USA

Mr. Paul H. Wright, Indianapolis, Indiana, USA 
The Princeton Plasma Physics Laboratory is operated by Princeton University under contract with the U.S. Department of Energy.

\author{
Information Services \\ Princeton Plasma Physics Laboratory \\ P.O. Box 451 \\ Princeton, NJ 08543
}

Phone: 609-243-2750

Fax: 609-243-2751

e-mail: pppl_info@pppl.gov

Internet Address: http://www.pppl.gov 